\title{
Clinical significance of CTHRC1 protein expression in human cancers: a meta-analysis
}

\author{
S.F. Wang ${ }^{1 *}$, Z. Yin ${ }^{2 *}$, J.J. Yin ${ }^{3}$, W. Zhang ${ }^{4}$ and C.G. Dong ${ }^{5}$ \\ ${ }^{1}$ Department of Pathology, Linyi People's Hospital of Shandong Province, \\ Linyi, China \\ ${ }^{2}$ Department of Ophthalmology, \\ Zoucheng People's Hospital of Shandong Province, Zoucheng, China \\ ${ }^{3}$ Health Management Center, Qingdao Haici Medical Treatment Group, \\ Qingdao, China \\ ${ }^{4}$ Department of Emergency, Qingdao Municipal Hospital, Qingdao, China \\ ${ }^{5}$ Department of Pathology, Yantai Mountain Hospital, Yantai, China \\ *These authors contributed equally to this study. \\ Corresponding author: C.G. Dong \\ E-mail: dongchenggong1016@yeah.net
}

Genet. Mol. Res. 15 (2): gmr.15027855

Received October 20, 2015

Accepted January 18, 2016

Published May 23, 2016

DOI http://dx.doi.org/10.4238/gmr.15027855

\begin{abstract}
The aim of this meta-analysis was to investigate the overall diagnostic and prognostic values of CTHRC1 expression in human cancer development. Based on the inclusion and exclusion criteria, 8 cohort studies were included in the meta-analysis. The data were extracted, and analyses were performed using a random-effects model. Summary odds ratios (ORs) and effect sizes (ESs) with 95\% confidence intervals (CIs) were calculated to assess the strength of the associations. A total of 1065 cancer patients from the 8 studies were included in the meta-analysis. The results revealed a positive correlation of CTHRC1 protein expression in tumors with tumor-node-metastasis (TNM) stage and with lymph node (LN) metastasis (TNM: OR = 2.98, $95 \% \mathrm{CI}=1.48-6.00, \mathrm{P}=0.002 ; \mathrm{LN}: \mathrm{OR}=4.26,95 \% \mathrm{CI}=1.88-9.67, \mathrm{P}$ $=0.001)$. CTHRC1 expression was higher in tumors with sizes $\geq 5 \mathrm{~cm}$
\end{abstract}


than in tumors with sizes $<5 \mathrm{~cm}(\mathrm{OR}=2.39,95 \% \mathrm{CI}=1.12-5.09, \mathrm{P}=$ 0.024). Patients with higher CTHRC1 expression had decreased overall survival $(\mathrm{OS})(\mathrm{ES}=1.78,95 \% \mathrm{CI}=1.23-2.33, \mathrm{P}<0.001)$ and poorer disease-free survival (DFS) $(\mathrm{ES}=1.71,95 \% \mathrm{CI}=1.11-2.31, \mathrm{P}<0.001)$. Disease-stratified analyses yielded significantly different estimates of CTHRC1 levels in the majority of the subgroups (all $\mathrm{P}<0.05$ ). In conclusion, increased CTHRC1 expression is associated with advanced TNM stage, increased LN metastasis and tumor size, and decreased OS and DFS, indicating that CTHRC1 may be a biomarker for prognosis of cancer patients.

Key words: CTHRC1; Protein expression; Human tumors; Meta-analysis; Cancer

\section{INTRODUCTION}

Tumors are caused when cells lose their normal growth regulation at the genetic level, which leads to the formation of clonally abnormal dysplastic lesions (Wang et al., 2012). Generally speaking, tumors can be divided into two different types, benign and malignant (Lowe et al., 2013). Malignant tumors, also known as cancer, are the main causes of mortality in developed countries, and are the second leading public health concern among developing countries (Percy et al., 2013). Cancers in adolescents and adults aged 15-39 years account for less than $10 \%$ of all new cancer diagnoses in developed countries, with incidence rates rising in individuals aged from 20 to 80 years (Haggar et al., 2012; Harding et al., 2012). Moreover, in Europe, the incidence and mortality of cancer play a critical role in both planning and estimating the influence of cancer control programs at the country and regional levels (Ferlay et al., 2013). The incidence of cancer and its prognosis may contribute to overall cancer prevalence, which is also affected by the aging of the population and increasing population growth rates (Crocetti et al., 2013; Mihajlović et al., 2013). During cancer progression, only a few detached cancer cells that leave the primary tumor and survive in circulation are required to seed metastases in distant organs; therefore, metastases remain the main cause of cancer-related deaths (Gorges and Pantel, 2013). In this process, new biologically active gene products are expressed, which promote cell migration and reduce the deposition of collagen (Mihajlović et al., 2013). In recent years, many researchers have postulated that collagen triple helix repeat containing-1 (CTHRC1), a protein associated with collagen synthesis, has a significant positive correlation with many malignancies, and moreover, promotes the occurrence and development of malignant tumors (Kim et al., 2013b).

CTHRC1 is a novel $28-\mathrm{kDa}$ cancer-secreted glycosylated protein that is highly conserved in lower chordates and higher mammals (Pyagay et al., 2005). CTHRC1 has been shown to be expressed mainly in neointimal smooth muscle cells and adventitial fibroblasts of balloon-injured vessels (Chen et al., 2013). Clinical findings have demonstrated that CTHRC1 has the ability to promote the proliferation of certain cells, and inhibit the synthesis of type I collagen ( $\mathrm{Li}$ et al., 2011). Moreover, it has been suggested that CTHRC1 may be largely involved in repairing damaged blood vessel tissues by limiting collagen matrix deposition and improving cell migration (Ip et al., 2011). Additionally, it been demonstrated that CTHRC1 can mediate the activities of members of the Rho GTPase family of proteins, including Rac1, 
RhoA, and Cdc42, which are important in regulating cell migration through their effects on cytoskeletal reorganization and cell adhesion activation (Park et al., 2013; Zhou et al., 2014). Recently, several studies have revealed that CTHRC1 is highly up-regulated in solid tumors, and may have a significant association with the pathogenic mechanisms of several human cancers, including hepatocellular carcinoma, gastric cancer, pancreatic cancer, and colorectal cancer (Kharaishvili et al., 2011; Ren et al., 2011; Wang et al., 2012). Furthermore, it has been suggested that CTHRC1 may play a significant role in enhancing the migration and invasion of tumor cells, thereby promoting metastasis, potentially through the activation and enhancement of promoter de-methylation, as well as by altering signaling pathways mediated by growth factor b1 (Chen et al., 2013; Liu et al., 2013). Moreover, recent results showed that CTHRC1 may be involved with enhancing cell proliferation and survival by activating the pathways of several vital signaling molecules, which may lead to cancer progression and metastasis (Tan et al., 2013). To date, a variety of findings have demonstrated that the overexpression of CTHRC1 is strongly associated with the development of some cancers (Kim et al., 2013a; Gu et al., 2014). However, other studies have reported outcomes that contradict the role of CTHRC1 in cancer progression (Zhou and Tong, 2011; Kim et al., 2013a). Because of these contradictions, here we performed a meta-analysis to investigate the correlations between CTHRC1 protein expression and gastric cancer (GC), colorectal cancer (CRC), breast cancer (BC), hepatocellular carcinoma (HCC), cervical cancer (CC), and esophageal squamous cell carcinoma (ESCC).

\section{MATERIAL AND METHODS}

\section{Data sources and keywords}

A comprehensive search in several electronic databases, including the Web of Science, PubMed, Embase, China National Knowledge Infrastructure (CNKI) databases, and the Cochrane Library was performed in order to obtain studies for inclusion in the meta-analysis. The search strategies included the combinations of keywords for all studies published through March 1st, 2014. The keyword combinations for the initial search were as follows: "CTHRC1 protein, human", "CTHRC1", "Cthrc1", "Collagen triple helix repeat containing-1", or "Collagen triple helix repeat containing 1", AND "Neoplasms", "Neoplasm", "Cancer", "Cancers", "carcinomas", "carcinoma", "tumor", "tumors", or "Neoplasms". Manual searches through the reference lists of the retrieved articles were also performed to identify additional studies for inclusion.

\section{Selection criteria}

Studies of patients with tumors that included CTHRC1 protein expression as a risk factor were evaluated. Eligible articles were identified based on the following detailed inclusion criteria: 1) only cohort studies that discussed the association between CTHRC1 protein expression and cancer were included; 2) all patients diagnosed with cancer were confirmed by histopathology indicating invasion occurrence as described (Van Cutsem et al., $2011)$; 3) the number of cancer cases included was provided; 4) the number of cases were more than 35; and 5) sufficient data for tumor-node-metastasis (TNM) stage, lymph node (LN) metastasis, and tumor size were provided. The following studies were excluded: 1) those on hereditary tumors; 2) those on haplotypes only; 3 ) those with patients who had been treated with anti-tumor therapy; and 4) case-control studies. 


\section{Data extraction}

Two independent investigators extracted data from identified studies on the basis of the rigorous selection criteria. Accordingly, the following necessary data were collected: first author, source of publication, country of origin, study type, study design, patient age, patient sex, year of publication, patient ethnicity, sample size, source of samples, detection methods, TNM stage, LN metastasis, tumor size, overall survival (OS), and disease-free survival (DFS). All of the authors of the selected studies approved to include their studies in this meta-analysis.

\section{Quality assessment}

The Newcastle-Ottawa Scale (NOS) criteria were utilized to evaluate the quality of each eligible study (Stang, 2010). The NOS criteria are presented as follows: 1) group selection: whether the exposure group has very good or better representation (NOS01); whether the non-exposure group and exposure group come from the same populations (NOS02); whether the enrolled studies have credible records or well-structured interviews (NOS03); whether the beginning of the study has no observational outcomes (NOS04); 2) group comparability: whether the study is in accordance with the most important factors to select and analyze the controls (NOS05); whether the study controls other important confounding factors (NOS06); 3) group results: whether the study is independent or belongs to a blind method (NOS07); whether the follow-up time is enough for the occurrence of results (NOS08); whether there are a small number of subjects losing to follow-up in the follow-up of all subjects that don't introduce deviation (NOS09). According to these criteria, studies with scores between 0 and 6 were considered of low quality, while those with scores between 7 and 9 were considered of high quality.

\section{Statistical analysis}

The STATA 12.0 software (Stata Corp, College Station, TX, USA) was used for all data analyses in the current study. Heterogeneity among studies was examined using a random-effects model, and a fixed-effects model was chosen when statistical heterogeneity was not present. Summary odds ratios (ORs) and effect sizes (ESs) with their 95\% confidence intervals (CI) were used for assessing the strength of associations, which were also confirmed by $\mathrm{Z}$ tests. The heterogeneity of subgroup meta-analyses and potential heterogeneity between studies were evaluated by the Cochran's Q -statistic where a value of $\mathrm{P}<0.05$ was deemed to be statistically significant, and an $\mathrm{I}^{2}$ test, respectively (Peters et al., 2006; Jackson et al., 2012). One-way sensitivity analysis was conducted for the pooled ORs of each eligible study to determine whether the results would be affected by the heterogeneity. Publication bias was assessed by funnel plot analysis as this may affect the validity, and Egger's linear regression test was conducted for further evaluation of the symmetry of the funnel plots (Zintzaras and Ioannidis, 2005). A P value of less than 0.05 was considered to be statistically significant.

\section{RESULTS}

\section{Characteristics of the included studies}

Figure 1 presents the PRISMA flow diagram of the screening steps used for the 82 
studies retrieved from the electronic databases. A total of 58 studies were excluded, including 2 duplicates; 12 letters, reviews, or meta-analyses; 20 non-human studies; and 24 studies that were not related to the research topics. An additional 14 trials were excluded after review of the full text, of which 2 were not case-control or cohort studies; 3 were not relevant to CTHRC1 protein expression; and 9 were not relevant to cancer. Following further review of the 10 remaining studies, 2 were abandoned due to lack of sufficient information. Therefore, 8 studies, which included a total of 1,065 cancer patients, met our rigorous inclusion criteria (Liu et al., 2009; Zhou and Tong, 2011; Wang et al., 2012; Chen et al., 2013; Kim et al., 2013a; Yin et al., 2013; Gu et al., 2014; Kim et al., 2014). The year of publication of the enrolled studies ranged from 2009 to 2014. All included studies were cohort studies that evaluated the correlation between CTHRC1 protein expression and cancer in Chinese (6 studies) and Korean (2 studies) populations. The cancer types include GC, CRC, BC, HCC, CC, and ESCC. The sources of the samples for CTHRC1 protein expression evaluation in the studies included in the meta-analysis were tissue biopsies. Enzyme linked immunosorbent assays (ELISA), streptavidin-peroxidase (SP), EnVision ELISA, and avidin-biotin peroxidase complexes (ABC) were used in the included studies to evaluate CTHRC1 protein expression. The NOS quality scores of all included studies were $>6$. A summary of the characteristics of the included studies is shown in Table 1 .

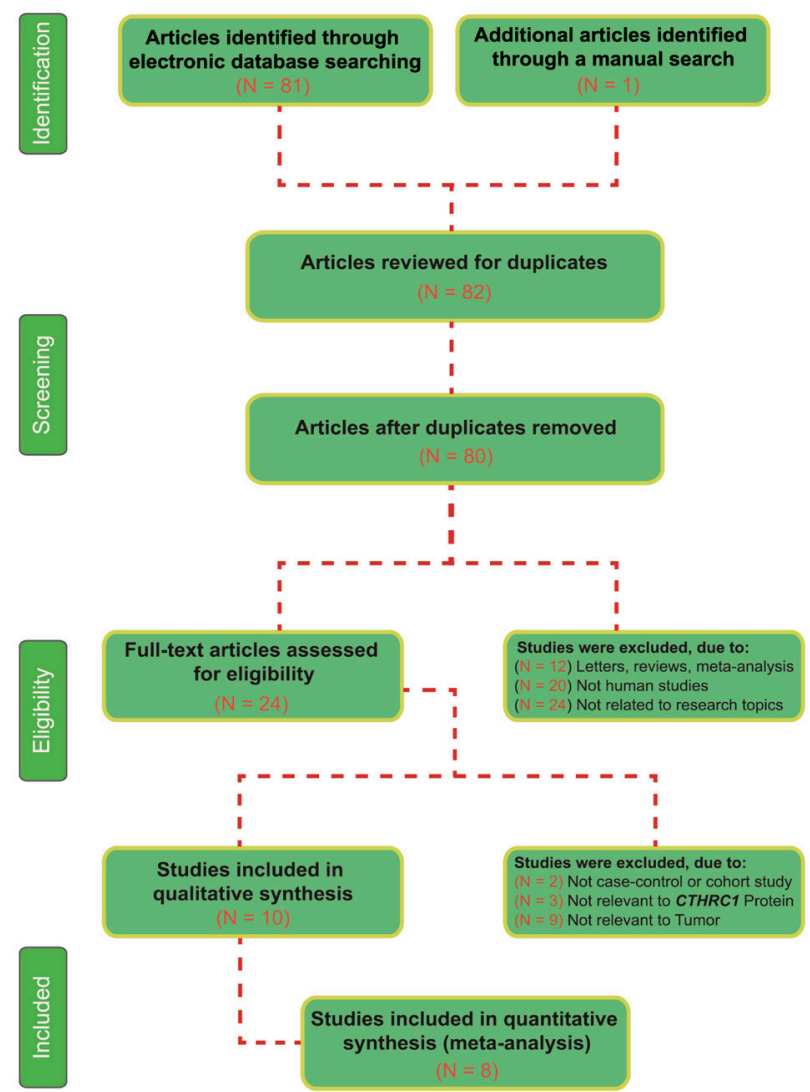

Figure 1. PRISMA flow diagram showing the study selection procedure. Eight cohort studies were included in the meta-analysis. 
Table 1. Characteristics of the included studies that focused on CTHRC1 protein expression in different cancers.

\begin{tabular}{l|l|l|l|c|c|c|c|l|l|l}
\hline First author & Year & Country & Ethnicity & Patients (N) & Gender (M/F) & Age (years) & Cancer & Sample & Method & NOS score \\
\hline Gu L & 2014 & China & Asians & 166 & $116 / 50$ & $59(33-81)$ & GC & Tissue & ABC & 8 \\
\hline Kim HC & 2014 & Korea & Asians & 188 & - & - & CRC & - & EnVision & 8 \\
\hline Yin WH & 2013 & China & Asians & 120 & $72 / 48$ & $59(23-86)$ & CRC & Tissue & SP & 8 \\
\hline Kim JH & 2013 & Korea & Asians & 189 & - & - & BC & Tissue & EnVision & 7 \\
\hline Chen YL & 2013 & China & Asians & 201 & $157 / 44$ & - & HCC & Tissue & SP & 8 \\
\hline Wang P & 2012 & China & Asians & 116 & - & - & GC & Tissue & SP & 7 \\
\hline Zhou Q & 2011 & China & Asians & 39 & $0 / 39$ & $40.5 \pm 8.3$ & CC & Tissue & SABC & 6 \\
\hline Liu W & 2009 & China & Asians & 46 & $35 / 11$ & - & ESCC & Tissue & SP & 6 \\
\hline
\end{tabular}

$\mathrm{CTHRC} 1=$ collagen triple helix repeat containing-1; $\mathrm{M}=$ male; $\mathrm{F}=$ female; $\mathrm{ABC}=$ avidin-biotin peroxidase complex; $\mathrm{SP}=$ streptavidin-peroxidase $; \mathrm{GC}=$ gastric cancer; $\mathrm{CRC}=$ colorectal cancer; $\mathrm{BC}=$ breast cancer; $\mathrm{HCC}=$ hepatocellular carcinoma; $\mathrm{CC}=$ cervical cancer; $\mathrm{ESCC}=$ esophageal squamous cell carcinoma; $\mathrm{NOS}=$ NewcastleOttawa scale.

\section{CTHRC1 protein expression in tumors}

A total of 8 cohort studies evaluated CTHRC1 protein expression in different types of tumors. The major results of the correlations between CTHRC1 protein expression and TNM stage, LN metastasis, and tumor size are presented in Figure 2. The random-effects model was used as heterogeneity was present $(\mathrm{P}<0.05)$. A positive association between CTHRC1 protein expression and TNM stage was identified by our meta-analysis $(\mathrm{OR}=2.98,95 \% \mathrm{CI}$ $=1.48-6.00, \mathrm{P}=0.002)$. It was also found that CTHRC1 protein expression was higher in tumors with $\mathrm{LN}$ metastasis than in tumors without $\mathrm{LN}$ metastasis $(\mathrm{OR}=4.26,95 \% \mathrm{CI}=1.88$ 9.67, $\mathrm{P}=0.001)$. CTHRC1 protein expression was observed to be higher in tumors with sizes $\geq 5 \mathrm{~cm}$ than in tumors with sizes $<5 \mathrm{~cm}(\mathrm{OR}=2.39,95 \% \mathrm{CI}=1.12-5.09, \mathrm{P}=0.024)$. Figure 3 shows the relationships between CTHRC1 protein expression and OS and DFS. The metaanalysis findings showed that patients with higher expression of CTHRC1 had decreased OS $(\mathrm{ES}=1.78,95 \% \mathrm{CI}=1.23-2.33, \mathrm{P}<0.001)$. Additionally, patients with higher expression of CTHRC1 had poorer DFS than patients with lower CTHRC1 expression (ES $=1.71,95 \% \mathrm{CI}$ $=1.11-2.31, \mathrm{P}<0.001)$.

Subgroup analyses based on cancer type demonstrated that high expression of CTHRC1 was positively associated with TNM stage in patients with GC, BC, and HCC $(\mathrm{P}<0.05)$, but no similar relationship was found in patients with CRC or CC (Figure 4). Subgroup analyses by cancer type also revealed that there was a positive association between CTHRC1 expression and LN metastasis in the BC and ESCC subgroups $(\mathrm{P}<0.05)$, whereas this association was not observed in the GC and $\mathrm{CC}$ subgroups. Furthermore, tumor sizes $\geq 5$ $\mathrm{cm}$ were correlated with high CTHRC1 expression in the BC and HCC subgroups $(\mathrm{P}<0.05)$, but not in the GC, CRC, or ESCC subgroups. Subgroup analyses by disease showed that high expression of CTHRC1 was related to OS in patients with CRC and HCC (both $\mathrm{P}<0.001$ ), but not in patients with GC or BC. Additionally, disease based subgroup analyses revealed that CTHRC1 protein expression was correlated to DFS in patients with $\mathrm{HCC}(\mathrm{P}<0.001)$, but not in patients with CRC or GC (Figure 4). Further subgroup analyses based on detection method showed there was an association between CTHRC1 protein expression and TNM stage and LN metastasis using non-SP and SP methods (all $\mathrm{P}<0.05$ ). Subgroup analyses based on detection method also revealed that CTHRC1 protein expression was strongly correlated with tumor size in the non-SP subgroups $(\mathrm{OR}=8.01,95 \% \mathrm{CI}=3.69-17.40, \mathrm{P}<0.001)$, but a similar correlation was not found in the SP subgroups (Figure 4). 


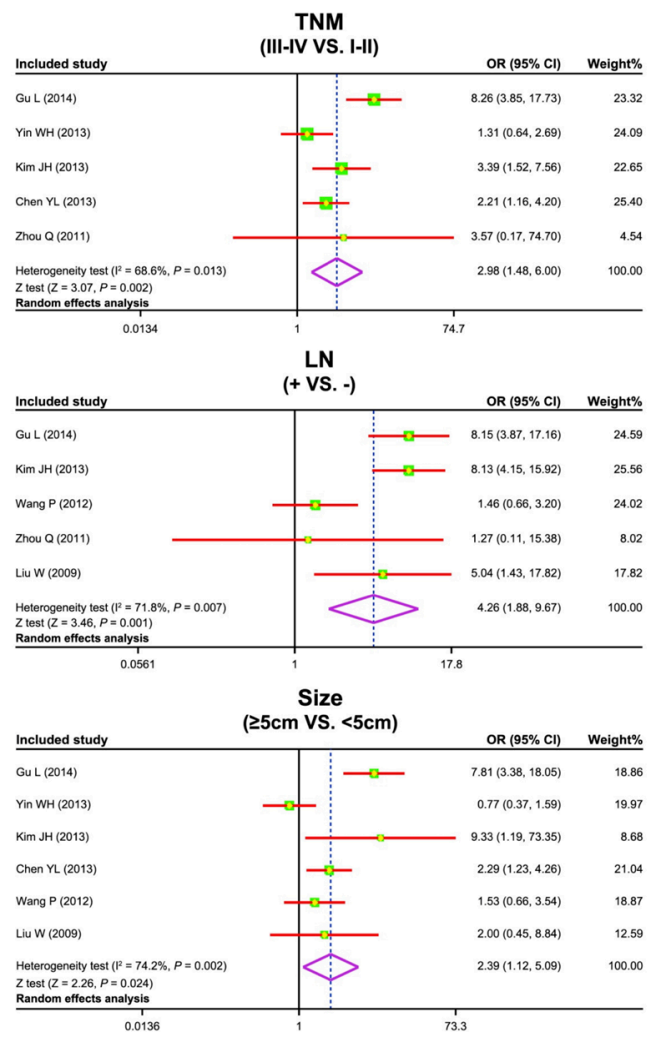

Figure 2. Forest plots and funnel plots of publication biases for the relationships between CTHRC1 protein expression and pathological characteristics of human tumors.

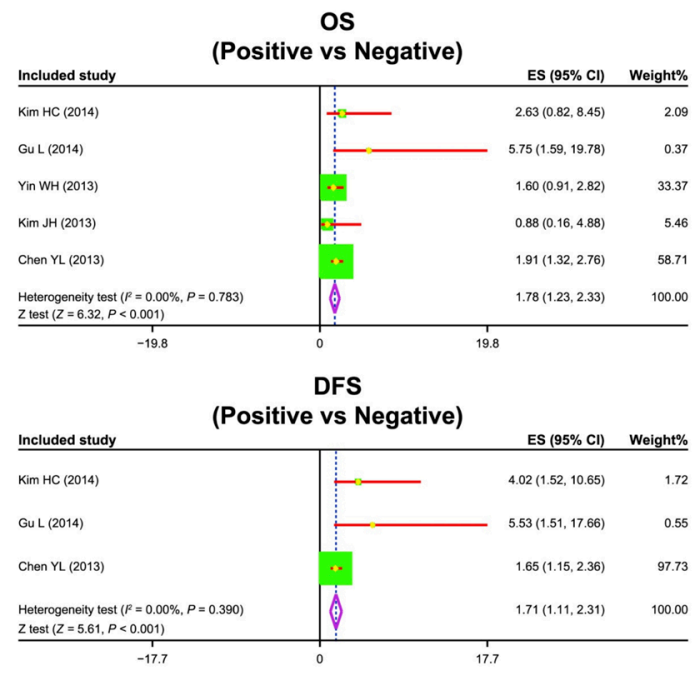

Figure 3. Forest plots and funnel plots of publication biases for the relationships between CTHRC1 expression and prognosis of patients with human tumors. 
Furthermore, subgroup analyses by detection method revealed a relationship between CTHRC1 protein expression and OS in the SP subgroups, but not in the non-SP subgroups. Additionally, CTHRC1 protein expression was found to be correlated with DFS in studies using either the SP or non-SP methods $(\mathrm{P}<0.05)$ (Figure 4).
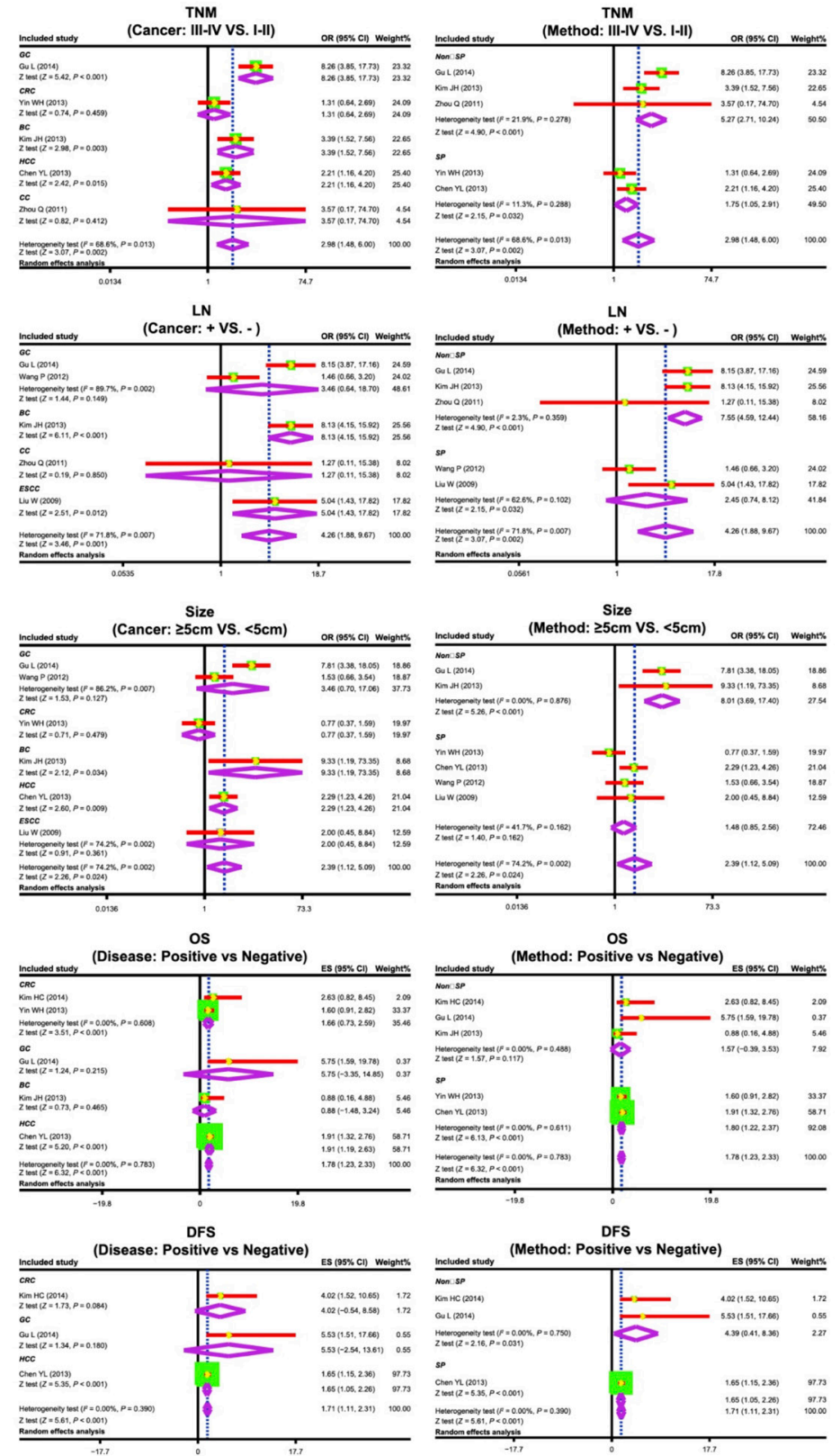

Figure 4. Subgroup analyses of the relationships between CTHRC1 protein expression and the pathological characteristics of tumors and prognosis of cancer patients. 


\section{Sensitivity analysis and publication bias}

Sensitivity analysis showed that no changes of statistical significance were found by removing any single study (Figures 5 and 6). Symmetrical graphs were observed among the 8 studies, and no publication bias was found in the associations between CTHRC1 protein expression and TNM stage, LN metastasis, tumor size, and OS by Egger's test (all P >0.05) (Figure 5,6). The funnel plot for the correlation of CTHRC1 protein expression with DFS of the 8 included studies showed slight asymmetry, and Egger's test revealed that publication bias existed $(\mathrm{P}=0.015)$ (Figure 6).
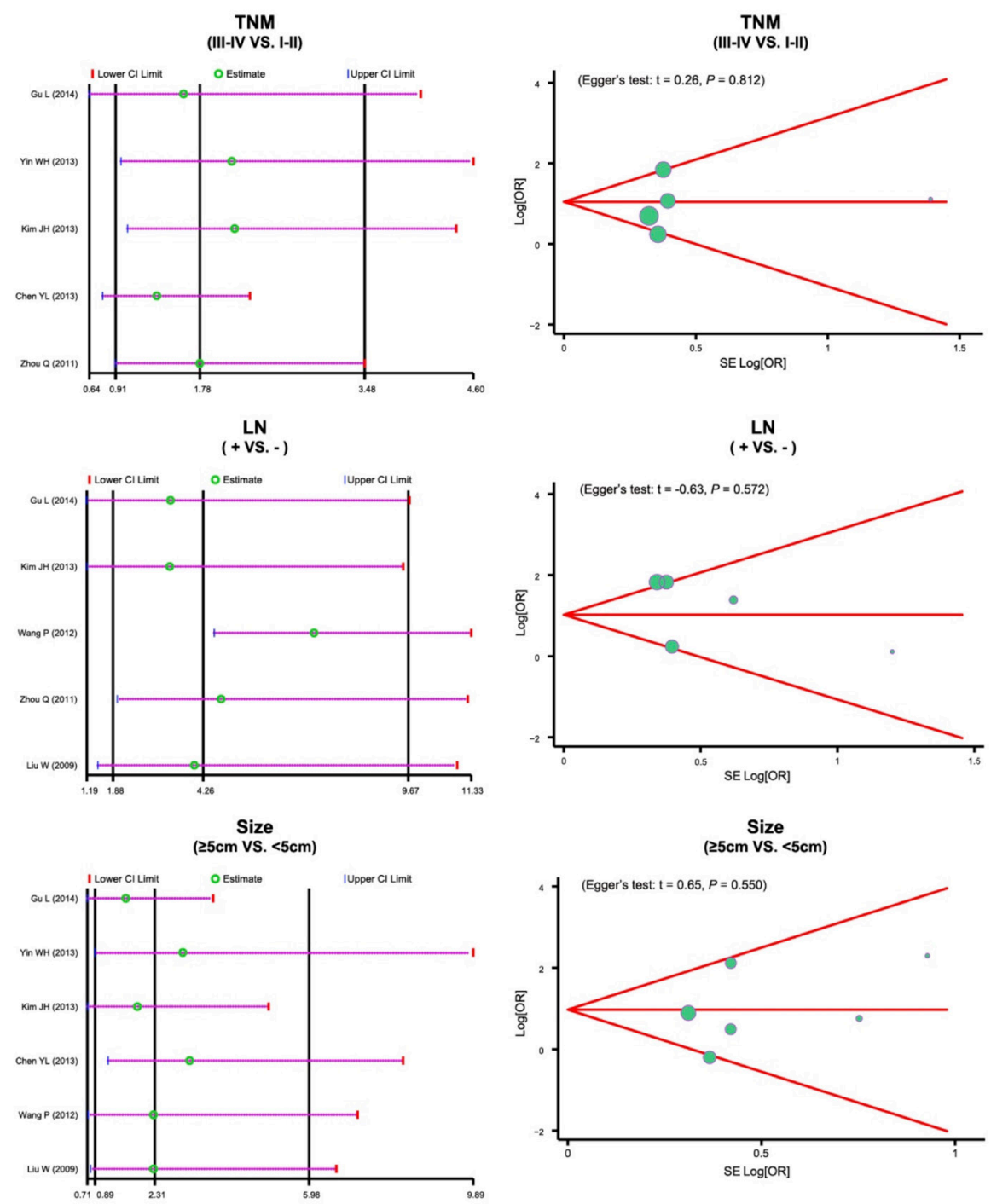

Figure 5. Sensitivity analyses of the summary odds ratio coefficients and funnel plots of publication biases for the associations between CTHRC1 protein expression and the pathological characteristics of human tumors. 

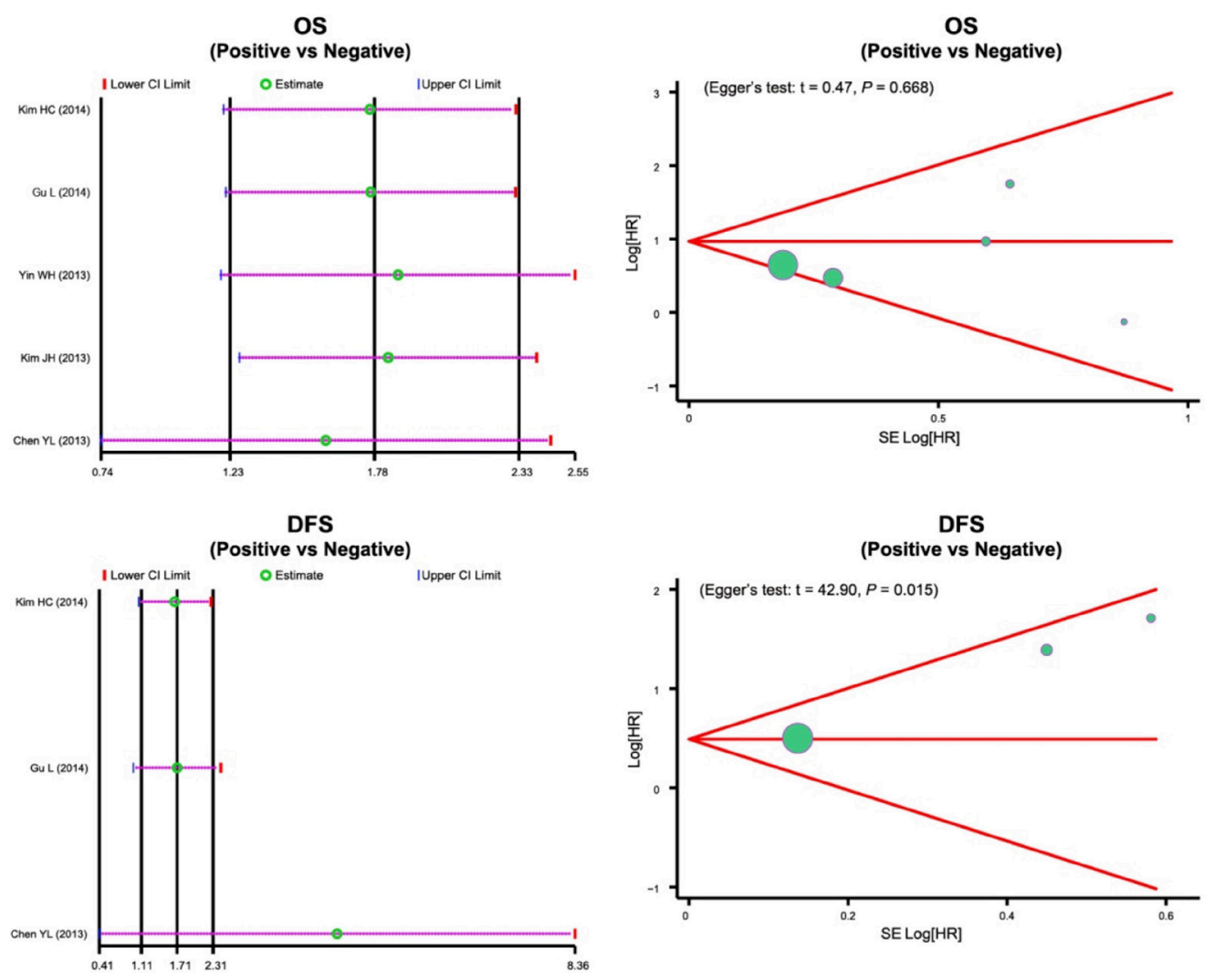

Figure 6. Sensitivity analyses of the summary odds ratio coefficients and funnel plots of publication biases for the associations between CTHRC1 protein expression and prognosis of cancer patients.

\section{DISCUSSION}

The current meta-analysis was performed in order to better understand the pathologic significance of CTHRC1 expression in human tumors and the connection between CTHRC1 expression and cancer prognosis. We found that CTHRC1 expression in the tumors of patients with stage III/IV cancers was higher than that in patients with stage I/II cancers, indicating that CTHRC1 expression may be involved in cancer progression. Nevertheless, the full impact of CTHRC1 expression on cancer has not yet been elucidated. CTHRC1 is widely accepted to be involved in vascular tissue repair and remodeling in response to injury by repressing the deposition of the collagen matrix and by facilitating the migration of cells (Pyagay et al., 2005). Additionally, CTHRC1 anchored in the cell membrane can stabilize the interaction of the Wnt-Frizzled receptor, and can selectively activate the PCP pathway, which was deemed as one part of Wnt signaling (Yamamoto et al., 2008). It is well understood that the Wnt/PCP signaling pathway controls tissue polarity and cell movement, and also participates in tumor dissemination and metastasis (Pukrop and Binder, 2008). In accordance with our findings, Kim et al. (2013a) demonstrated that over-expression of CTHRC1 is be closely related to nodal metastasis and TNM stage in BC patients, indicating that the expression of CTHRC1 
may be an important factor in tumor progression and tumor cell migration, and thus may be useful in the prediction of clinical outcomes of cancer patients.

Our results also revealed that CTHRC1 expression in the tumors of patients with positive LN status and tumor sizes of $5 \mathrm{~cm}$ or larger was higher than that in the tumors of patients with negative LN status and tumor sizes smaller than $5 \mathrm{~cm}$, indicating that CTHRC1 expression may be related to tumor metastasis and tumor cell proliferation. CTHRC1 is considered active and potent in extracellular matrix (ECM) protein degradation ( $\mathrm{Li}$ et al., 2011). In the tumor micro-environment, the ECM, which consists of the basement membrane and interstitial connective tissue, has been shown to play a vital role in the processes of proliferation, migration, and metastasis of tumor cells (Kim et al., 2011). Consequently, we hypothesize that aberrant CTHRC1 expression may give rise to the development of tumors in humans. Another important finding of the current meta-analysis was that cancer patients with positive CTHRC1 expression exhibited shorter OS and DFS than those that were CTHRC1 negative, implying that increased expression of CTHRC1 in tumors may contribute to poorer prognosis for cancer patients. This could be explained by the observation that increased secretion of CTHRC1 by tumor and stromal cells can stimulate tumor progression and LN metastasis by inducing alterations in the structural composition of the stromal ECM in the tumor micro-environment (Kim et al., 2013a). A previous study by Gu et al. (2014) provided evidence to support the notion that high expression of CTHRC1 has a strong impact on the progression of gastric carcinoma, and is significantly linked to poor prognosis, which was observed independently of other factors.

Additional stratified analyses that focused on cancer type and detection method were also carefully performed in an attempt to understand the correlations of CTHRC1 expression with pathological characteristics and prognosis of cancers more precisely and profoundly. In the cancer-type based subgroup analyses, the results revealed that CTHRC1 expression was correlated with TNM stage in GC, BC, and HCC, but not in CRC or CC. Furthermore, CTHRC1 expression was found to be associated with LN metastasis in BC and ESCC, but not in $\mathrm{GC}$ or $\mathrm{CC}$. We also observed that the expression of CTHRC1 may be linked to tumor size in $\mathrm{BC}$ and $\mathrm{HCC}$, while there was no such relationship between CTHRC1 expression and tumor size in GC, CRC, or ESCC. In regards to cancer prognosis, our results indicated that CTHRC1 expression was associated with OS in CRC and HCC, whereas it was only associated with DFS in HCC. Additionally, higher CTHRC1 expression appeared to contribute to poorer prognoses in the majority of the cancer subgroups. Taken together, the main findings of the current study were in agreement with previous studies, and suggest that CTHRC1 expression may be correlated with pathological characteristics of certain cancers, and moreover, may be utilized to predict the prognosis of cancer patients.

There were some limitations in the present study. First, the existence of publication bias could have influenced our results. Second, the selected studies were written only in Chinese or English, and thus the absence of articles written in other languages may have also caused bias to the current meta-analysis. Finally, publication bias was detected in the Egger's test for the correlation of CTHRC1 expression with DFS, indicating that the resultant data may not reflect the actual relationship. In spite of these aforementioned limitations, the significant findings revealed by our meta-analysis should be ignored, which shed light on the involvement of CTHRC1 in the development and progression of human cancers.

In summary, our data strongly suggest that high CTHRC1 expression is correlated with TNM stage, LN metastasis, and tumor size, as well as poor OS and DFS in different 
cancers. The crucial role of CTHRC1 in human cancer progression may make it conducive for clinical utility in predicting the prognosis of cancer patients.

\section{Conflicts of interest}

The authors have declared that no conflict of interest exist.

\section{ACKNOWLEDGMENTS}

We would like to acknowledge the reviewers for their helpful comments regarding this work.

\section{REFERENCES}

Chen YL, Wang TH, Hsu HC, Yuan RH, et al. (2013). Overexpression of CTHRC1 in hepatocellular carcinoma promotes tumor invasion and predicts poor prognosis. PLoS One 8: e70324. http://dx.doi.org/10.1371/journal.pone.0070324

Crocetti E, De Angelis R, Buzzoni C, Mariotto A, et al.; AIRTUM Working group (2013). Cancer prevalence in United States, Nordic Countries, Italy, Australia, and France: an analysis of geographic variability. Br. J. Cancer 109: 219228. http://dx.doi.org/10.1038/bjc.2013.311

Ferlay J, Steliarova-Foucher E, Lortet-Tieulent J, Rosso S, et al. (2013). Cancer incidence and mortality patterns in Europe: estimates for 40 countries in 2012. Eur. J. Cancer 49: 1374-1403. http://dx.doi.org/10.1016/j.ejca.2012.12.027

Gorges TM and Pantel K (2013). Circulating tumor cells as therapy-related biomarkers in cancer patients. Cancer Immunol. Immunother. 62: 931-939. http://dx.doi.org/10.1007/s00262-012-1387-1

Gu L, Liu L, Zhong L, Bai Y, et al. (2014). Cthrc1 overexpression is an independent prognostic marker in gastric cancer. Hum. Pathol. 45: 1031-1038. http://dx.doi.org/10.1016/j.humpath.2013.12.020

Haggar FA, Preen DB, Pereira G, Holman CD, et al. (2012). Cancer incidence and mortality trends in Australian adolescents and young adults, 1982-2007. BMC Cancer 12: 151.http://dx.doi.org/10.1186/1471-2407-12-151

Harding C, Pompei F and Wilson R (2012). Peak and decline in cancer incidence, mortality, and prevalence at old ages. Cancer 118: 1371-1386. http://dx.doi.org/10.1002/cncr.26376

Ip W, Wellman-Labadie O, Tang L, Su M, et al. (2011). Collagen triple helix repeat containing 1 promotes melanoma cell adhesion and survival. J. Cutan. Med. Surg. 15: 103-110. http://dx.doi.org/10.2310/7750.2011.10014

Jackson D, White IR and Riley RD (2012). Quantifying the impact of between-study heterogeneity in multivariate metaanalyses. Stat. Med. 31: 3805-3820.http://dx.doi.org/10.1002/sim.5453

Kharaishvili G, Cizkova M, Bouchalova K, Mgebrishvili G, et al. (2011). Collagen triple helix repeat containing 1 protein, periostin and versican in primary and metastatic breast cancer: an immunohistochemical study. J. Clin. Pathol. 64: 977-982. http://dx.doi.org/10.1136/jclinpath-2011-200106

Kim HC, Kim YS, Oh HW, Kim K, et al. (2014). Collagen triple helix repeat containing 1 (CTHRC1) acts via ERKdependent induction of MMP9 to promote invasion of colorectal cancer cells. Oncotarget 5: 519-529. http://dx.doi. org/10.18632/oncotarget.1714

Kim JH, Baek TH, Yim HS, Kim KH, et al. (2013a). Collagen triple helix repeat containing-1 (CTHRC1) expression in invasive ductal carcinoma of the breast: the impact on prognosis and correlation to clinicopathologic features. Pathol. Oncol. Res. 19: 731-737. http://dx.doi.org/10.1007/s12253-013-9636-y

Kim MJ, Kang YU, Kim CS, Choi JS, et al. (2013b). Proteinuria as a risk factor for mortality in patients with colorectal cancer. Yonsei Med. J. 54: 1194-1201.http://dx.doi.org/10.3349/ymj.2013.54.5.1194

Kim SH, Turnbull J and Guimond S (2011). Extracellular matrix and cell signalling: the dynamic cooperation of integrin, proteoglycan and growth factor receptor. J. Endocrinol. 209: 139-151. http://dx.doi.org/10.1530/JOE-10-0377

Li J, Cao J, Li M, Yu Y, et al. (2011). Collagen triple helix repeat containing-1 inhibits transforming growth factor-b1induced collagen type I expression in keloid. Br. J. Dermatol. 164: 1030-1036. http://dx.doi.org/10.1111/j.13652133.2011.10215.x

Liu G, Sengupta PK, Jamal B, Yang HY, et al. (2013). N-glycosylation induces the CTHRC1 protein and drives oral cancer cell migration. J. Biol. Chem. 288: 20217-20227.http://dx.doi.org/10.1074/jbc.M113.473785

Liu WSR, Zhu H and Hao B (2009). ITGA6, CTHRC1 expression in esophageal squamous cell cancer and their clinical significance. Acta Universitatis Medicinalis Nanjing 29: 508-511. 
Lowe KA, Chia VM, Taylor A, O’Malley C, et al. (2013). An international assessment of ovarian cancer incidence and mortality. Gynecol. Oncol. 130: 107-114.http://dx.doi.org/10.1016/j.ygyno.2013.03.026

Mihajlović J, Pechlivanoglou P, Miladinov-Mikov M, Zivković S, et al. (2013). Cancer incidence and mortality in Serbia 1999-2009. BMC Cancer 13: 18. http://dx.doi.org/10.1186/1471-2407-13-18

Park EH, Kim S, Jo JY, Kim SJ, et al. (2013). Collagen triple helix repeat containing-1 promotes pancreatic cancer progression by regulating migration and adhesion of tumor cells. Carcinogenesis 34: 694-702. http://dx.doi. org/10.1093/carcin/bgs378

Percy AJ, Chambers AG, Yang J and Borchers CH (2013). Multiplexed MRM-based quantitation of candidate cancer biomarker proteins in undepleted and non-enriched human plasma. Proteomics 13: 2202-2215. http://dx.doi. org/10.1002/pmic.201200316

Peters JL, Sutton AJ, Jones DR, Abrams KR, et al. (2006). Comparison of two methods to detect publication bias in metaanalysis. JAMA 295: 676-680. http://dx.doi.org/10.1001/jama.295.6.676

Pukrop T and Binder C (2008). The complex pathways of Wnt 5a in cancer progression. J. Mol. Med. 86: 259-266. http:// dx.doi.org/10.1007/s00109-007-0266-2

Pyagay P, Heroult M, Wang Q, Lehnert W, et al. (2005). Collagen triple helix repeat containing 1, a novel secreted protein in injured and diseased arteries, inhibits collagen expression and promotes cell migration. Circ. Res. 96: 261-268. http://dx.doi.org/10.1161/01.RES.0000154262.07264.12

Ren N, Wu JC, Dong QZ, Sun HJ, et al. (2011). Association of specific genotypes in metastatic suppressor HTPAP with tumor metastasis and clinical prognosis in hepatocellular carcinoma. Cancer Res. 71: 3278-3286. http://dx.doi. org/10.1158/0008-5472.CAN-10-3100

Stang A (2010). Critical evaluation of the Newcastle-Ottawa scale for the assessment of the quality of nonrandomized studies in meta-analyses. Eur. J. Epidemiol. 25: 603-605. http://dx.doi.org/10.1007/s10654-010-9491-z

Tan F, Liu F, Liu H, Hu Y, et al. (2013). CTHRC1 is associated with peritoneal carcinomatosis in colorectal cancer: a new predictor for prognosis. Med. Oncol. 30: 473. http://dx.doi.org/10.1007/s12032-013-0473-3

Van Cutsem E, Dicato M, Geva R, Arber N, et al. (2011). The diagnosis and management of gastric cancer: expert discussion and recommendations from the 12th ESMO/World Congress on Gastrointestinal Cancer, Barcelona, 2010. Ann. Oncol. 22 (Suppl 5): v1-v9. http://dx.doi.org/10.1093/annonc/mdr284

Wang P, Wang YC, Chen XY, Shen ZY, et al. (2012). CTHRC1 is upregulated by promoter demethylation and transforming growth factor-b1 and may be associated with metastasis in human gastric cancer. Cancer Sci. 103: 1327-1333.http:// dx.doi.org/10.1111/j.1349-7006.2012.02292.x

Yamamoto S, Nishimura O, Misaki K, Nishita M, et al. (2008). Cthrc1 selectively activates the planar cell polarity pathway of Wnt signaling by stabilizing the Wnt-receptor complex. Dev. Cell 15: 23-36. http://dx.doi.org/10.1016/j. devcel.2008.05.007

Yin WH, Fan HZ, Sheng JW, Xia HM, et al. (2013). Effect of vascular endothelial growth factor C and collagen triple helix repeat containing 1 expression on prognosis of rectal carcinoma patients. Zhonghua Wei Chang Wai Ke Za Zhi 16: 673-675.

Zhou QZW and Tong YQ (2011). Expression and clinical significance of collagen triple helix repeat containing 1 in cervical cancer. J. Chin. Pract. Diagn. Ther. 25: 677-679, 683.

Zhou S, Gao R, Hu W, Qian T, et al. (2014). MiR-9 inhibits Schwann cell migration by targeting Cthrc1 following sciatic nerve injury. J. Cell Sci. 127: 967-976. http://dx.doi.org/10.1242/jcs.131672

Zintzaras E and Ioannidis JP (2005). HEGESMA: genome search meta-analysis and heterogeneity testing. Bioinformatics 21: 3672-3673. http://dx.doi.org/10.1093/bioinformatics/bti536 